\title{
NON-HERMITIAN STAR-SHAPED QUANTUM GRAPHS
}

\author{
MiloslaV ZnOJIL* \\ Nuclear Physics Institute ASCR, 25068 Řež, Czech Republic \\ * corresponding author: znojil@ujf.cas.cz \\ ABstract. A compact review is given, and a few new numerical results are added to the recent studies \\ of the $q$-pointed one-dimensional star-shaped quantum graphs. These graphs are assumed endowed \\ with certain specific, manifestly non-Hermitian point interactions, localized either in the inner vertex \\ or in all of the outer vertices and carrying, in the latter case, an interesting zero-inflow interpretation.
}

KEYWORDS: quantum observables, hidden Hermiticity, non-local inner products, smearing requirement, discretized coordinates, exactly solvable quantum graphs.

\section{ThE CONCEPT OF QUANTUM GRAPHS}

The scope of the traditional quantum theory is currently being broadened to cover not only the $1 \mathrm{D}$ motion of (quasi)particles, say, along a single thin wire but also along the more complicated one-dimensional graph-shaped structures $\mathbb{G}$ which are, by definition, composed of some $N_{W}$ (finite or semi-infinite) wedges which may - though need not - be connected at some of the elements of the given set of $N_{V}$ vertices. Under the influence of the contemporary solid-state technologies these structures may be realized as ultrathin 1D-like waveguides with nontrivial topological, geometrical and/or physical properties. Nowadays, there exists an extensive literature on the subject (cf., e.g., [1]).

From the formal point of view, one of the key features of these structures is the necessity of their description using the formalism of Quantum Mechanics. This necessity opens, naturally, a number of challenging questions which concern both the kinematics (for this reason, in a typical quantum graph model one assumes a free motion along the interior of the edges) and the dynamics (most often, one uses just point interactions which are localized strictly at the vertices).

\section{THE CONCEPT OF CRYPTO-HERMITICITY}

One of the most interesting mathematical questions addressed in the quantum-graph context concerns the proofs or the sufficient conditions of the self-adjoint nature of the corresponding Hamiltonian $H=H^{(\mathbb{G})}$ in a pre-selected, sufficiently "friendly" Hilbert space $\mathcal{H}^{(F)}$ of states. Recently, a new approach to this type of problems (carrying a nickname of $\mathcal{P} \mathcal{T}$-symmetric Quantum Mechanics) has been advocated and made popular by Carl Bender with multiple coauthors (cf. review [2]).

The mathematical essence of their recommendation may be seen in the replacement of the a priori selected friendly or "first" Hilbert space $\mathcal{H}^{(F)}$ by another, ad hoc and Hamiltonian-dependent "second" Hilbert space $\mathcal{H}^{(S)}$ which only differs from $\mathcal{H}^{(F)}$ by a redefinition of the inner product defined in terms of an operator $\Theta$ called "Hilbert space metric",

$$
\langle\phi \mid \psi\rangle^{(S)}:=\langle\phi|\Theta| \psi\rangle^{(F)} \equiv\left\langle\langle\phi \mid \psi\rangle^{(F)}\right.
$$

(we use the Dirac-like notation conventions as introduced and used in compact reviews [3 [5]).

As an immediate consequence one reveals, in general, that one may define a generic quantum system by a doublet of operators (i.e., by the Hamiltonian $H$ and metric $\Theta$ ) which only have to satisfy the following generalized version of the standard and necessary Hermiticity requirement

$$
H^{\dagger} \Theta=\Theta H, \quad 0<\Theta=\Theta^{\dagger}<\infty,
$$

which may be called a "disguised" Hermiticity or crypto-Hermiticity [6. The introduction of this concept may be attributed, historically, to Dieudonné [7] and, in the context of (nuclear) physics, to Scholtz et al [8].

\section{The CONCEPT OF CRYPTO-HERMITIAN QUANTUM GRAPHS}

Even for the most trivial, $N_{W}=1$ quantum graphs with $\mathbb{G}=\mathbb{R}$ it is by far not easy to satisfy the selfconsistency condition (2). In essence, two technical problems are being encountered. Firstly, we must guarantee that the energy spectrum of our Hamiltonian is real (this is necessary for probabilistic interpretation/tractability but, generically, the proof is difficult - see [9] for an illustrative example).

Secondly, the compatibility (2) between $H$ and $\Theta$ (studied, most often, as a brute-force construction of $\Theta=\Theta(H)$ ) is usually not an easy task, either (for the above-mentioned example, just various tedious approximative constructions may be found in the literature — cf. [10]). 


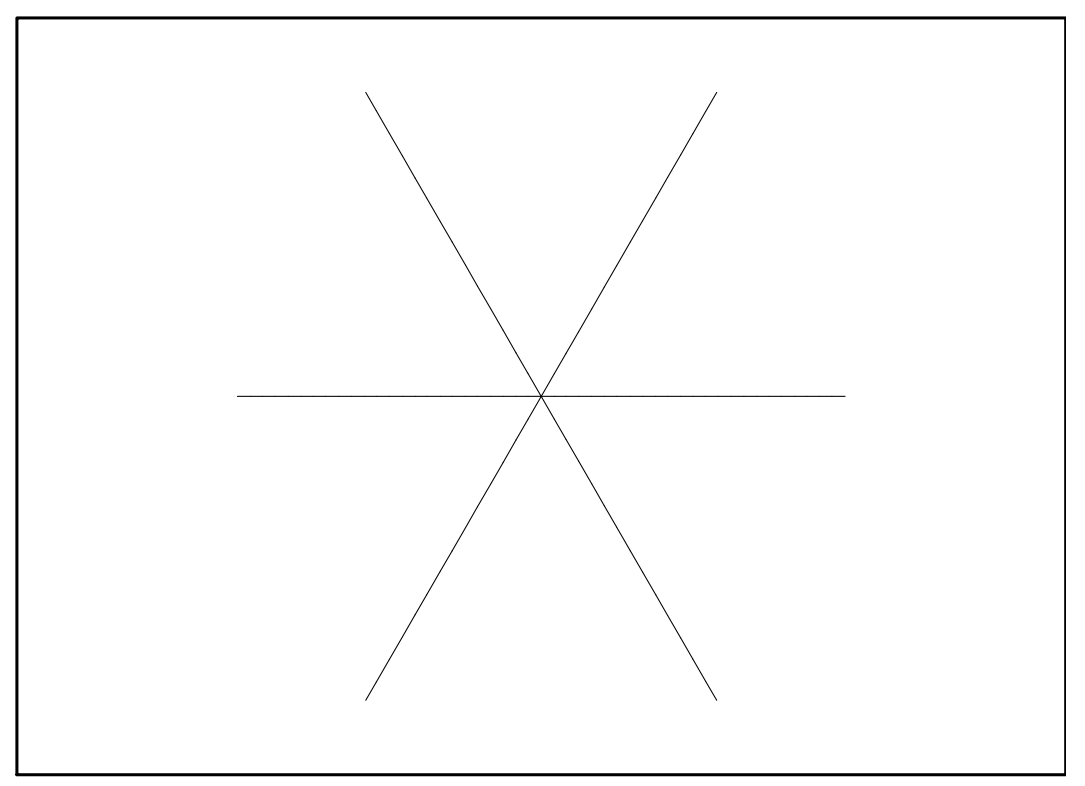

Figure 1. A typical six-pointed star graph.

This being said, one of the most efficient ways of addressing these technical challenges has been described in [11] and generalized, to nontrivial graphs, in its sequel [12. The guiding idea lied in the discretization of the graphs (see also 13] for a deeper analysis and discussion of this technique). Its use opened also the successful transition to the description of some topologically nontrivial graphs in 14 .

Unfortunately, for the topologically more complicated crypto-Hermitian graphs, the advantages of the discretization technique (which, basically, converted the underlying Schrödinger equation into a finite-dimensional matrix problem) proved more or less lost due to the growth of the difficulties caused by the necessity of finding a suitable metric $\Theta=\Theta(H)$.

In this sense, the discrete elementary solvable examples as described in [11, 12] appeared exceptional and exceptionally friendly. For this reason we returned, recently, to the study of the continuous quantum graphs again. In order to keep them tractable nonnumerically, we restricted our attention to the mere topologically trivial, star-shaped graphs in [15. Here we intend to recall one of these models and add a few comments, yielding the conclusion that the study of the $\mathcal{P} \mathcal{T}$-symmetric quantum graphs may be made sufficiently friendly not only via the simplification of kinematics (i.e., by the discretization of the wedges) but also via the alternative extreme simplification of dynamics. Not realized by the selection of the sufficiently elementary form of the graph itself but rather by the use of the symmetry-rich point interactions at the vertices.

\section{The SIMPLIFICATIONS OF KINEMATICS}

During the study of the broad variety of the graphsrelated open questions one often selects the models where the underlying mathematics is simplified, to its very core, by the replacement of the real-world problem in $3 \mathrm{D}$ by its idealized representation by a piecewise linear 1D graph, on the wedges of which the Hamiltonian is represented by the Laplacean, $H \sim$ $-\triangle$. In such a scenario one may feel inspired by the simplest systems which live on the single straight line which may be finite (then, the system is, in effect, a square well possessing just bound states) or infinite (then, one usually speaks about the one-dimensional problem of scattering).

In the most natural direction of a generalization of the theory one then glues a $q$-plet of half-lines in a single vertex and arrives at the star-shaped family of graphs $\mathbb{G}^{(q)}$ (see Fig. 1 where $q=6$ and where all of the wedge lengths and angular distances of neighbors are equal).

Naturally, the main principles of Quantum Mechanics remain unchanged. In particular, the bound states living on a given star graph $\mathbb{G}^{(q)}$ will be represented by the quadratically integrable wave functions $\psi_{n}(x) \in L^{2}(\mathbb{G})$,

$$
\int_{\mathbb{G}} d x \psi_{n}^{*}(x) \psi_{n}(x)<\infty .
$$

Whenever the corresponding friendly and trivial metric $\Theta^{(F)}=I$ is replaced by its nontrivial version defining the correct physical Hilbert space $\mathcal{H}^{(S)}$, the "naive" Eq. (3) must be replaced by the double integral which defines, by the way, also the correct physical orthonormalization of the bound states,

$$
\int_{\mathbb{G}} d x \int_{\mathbb{G}} d x^{\prime} \psi_{m}^{*}(x) \Theta_{\left(x, x^{\prime}\right)} \psi_{n}\left(x^{\prime}\right)=\delta_{m n}
$$

where $\delta_{m n}$ is Kronecker symbol. For the Hamiltonians which are Hermitian in $\mathcal{H}^{(F)}$ the use of the so called Dirac's metric $\Theta_{\left(x, x^{\prime}\right)}=\delta\left(x-x^{\prime}\right)$ leads to the degenerate form (3) of Eq. (4) of course. 


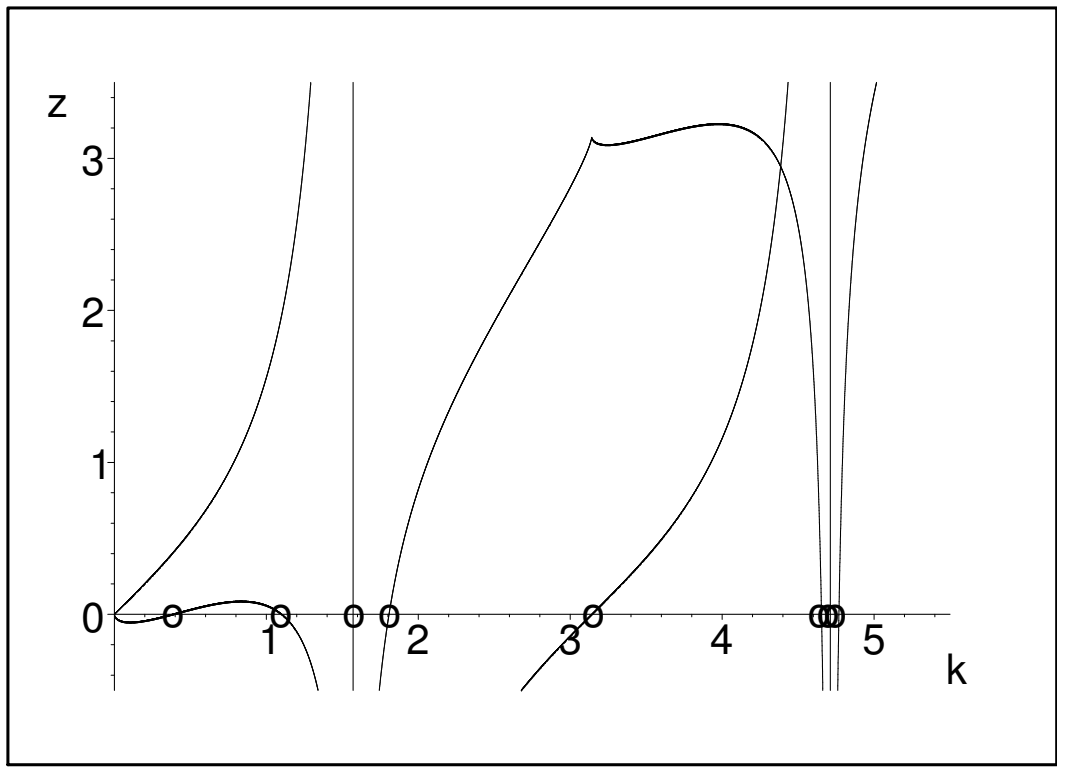

FiguRE 2. The real-momentum-dependence of the two factors of the secular determinant of Eq. 10 in the subcritical non-Hermitian regime with $\alpha=0.7$.

In the most natural move towards a more realistic scenario one often tries to think about a nonzero thickness of the wedges. Of course, with the necessary transition to the partial-differential Schrödinger equations this would make the eigenvalue problem highly nontrivial even for the straight-line 1D graph. In 11, an alternative idea of the smearing of the 1D wedges has been proposed, therefore. Its realization relied heavily upon the discretization of the 1D graph which reduced Eq. (2) to the mere $N$ by $N$ matrix problem. The key idea lied in the smearing of the wedges caused by the use of the smearing-providing metric $\Theta$. Illustrative examples were constructed in the form of the tridiagonal (or block-tridiagonal or block-pentadiagonal, etc) matrices $\Theta$ (see also [16] for a more detailed account of this metric-mediated smearing idea as well as of its possible phenomenological interpretation and consequences).

\section{The SIMPLIFICATIONS OF DYNAMICS}

The discretization of the star-shaped graphs rendered it possible to construct the smearing-metric matrices $\Theta$ for various models of dynamics. For illustration, we may recall the one-parametric toy-model discretegraph Hamiltonian of [11] with $N=4$,

$$
H^{(4)}(\lambda)=\left[\begin{array}{cccc}
2 & -1 & 0 & 0 \\
-1 & 2 & -1-\lambda & 0 \\
0 & -1+\lambda & 2 & -1 \\
0 & 0 & -1 & 2
\end{array}\right],
$$

which has been assigned there the four-parametric set of metrics

$$
\begin{aligned}
\Theta^{(4)}(\lambda)= & \Theta_{\left[\alpha_{1}, \alpha_{2}, \alpha_{3}, \alpha_{4}\right]}^{(4)}(\lambda) \\
& =\alpha_{1} M_{1}+\alpha_{2} M_{2}+\alpha_{3} M_{3}+\alpha_{4} M_{4},
\end{aligned}
$$

where

$$
\begin{gathered}
M_{1}=\left[\begin{array}{cccc}
1-\lambda & 0 & 0 & 0 \\
0 & 1-\lambda & 0 & 0 \\
0 & 0 & 1+\lambda & 0 \\
0 & 0 & 0 & 1+\lambda
\end{array}\right], \\
M_{2}=\left[\begin{array}{cccc}
0 & 1-\lambda & 0 & 0 \\
1-\lambda & 0 & 1-\lambda^{2} & 0 \\
0 & 1-\lambda^{2} & 0 & 1+\lambda \\
0 & 0 & 1+\lambda & 0
\end{array}\right], \\
M_{3}=\left[\begin{array}{cccc}
0 & 0 & 1 & 0 \\
0 & 1-\lambda & 0 & 1 \\
1 & 0 & 1+\lambda & 0 \\
0 & 1 & 0 & 0
\end{array}\right] \\
M_{4}=\left[\begin{array}{cccc}
0 & 0 & 0 & 1 \\
0 & 0 & 1 & 0 \\
0 & 1 & 0 & 0 \\
1 & 0 & 0 & 0
\end{array}\right]
\end{gathered}
$$

Obviously, in the light of the well known spectralexpansion formula [17] the general $N$ by $N$ matrix of the metric contains $N$ free real parameters so that due to the independence of components (7) the solution (6) of Eq. (2) is exhaustive whenever all the four $\alpha_{j}$ s are positive and whenever $|\lambda|<1$. Moreover, the first component $M_{1}$ is diagonal (so that we may conclude that the energy spectrum remains real). At the same time, merely the last component cannot be treated as a smearing-mediating band matrix.

Analogous (though, naturally, not so easily displayed) results were obtained, in [12], for the nontrivial discrete star graphs with $q>2$. In place of the diagonal, tridiagonal, pentadiagonal (etc) matrices (cf Eq. (7)) we merely had to construct there the blockdiagonal, block-tridiagonal, block-pentadiagonal (etc) matrices, respectively. 


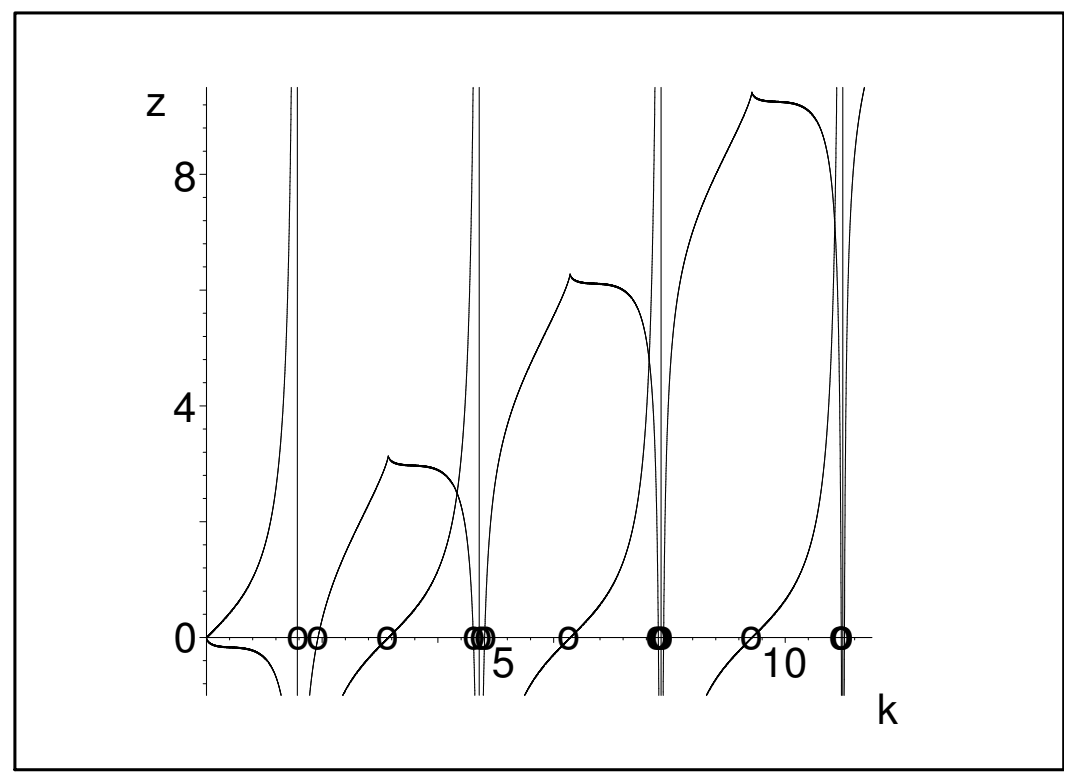

Figure 3. The real-momentum-dependence of the two factors of the secular determinant of Eq. 10 in the strongly non-Hermitian regime with $\alpha=1$.

\section{An illustrative, EXACTLY SOLVABLE STAR-SHAPED 1D QUANTUM GRAPH}

The main technical disadvantage of the discretization recipe lies in the quick weakening of the merits (viz., of the tractability of the mathematics by means of linear algebra) with the growth of $q$ and/or of the matrix dimensions (say, beyond $N>10$ [12]). The occurrence of these limitations forced us to return, recently, to the continuous graphs of the star-shaped form which is sampled in Fig. 1. In [15], in particular, we omitted the "central-vertex" interaction (as used in [12]) completely. This means that just the most elementary Kirchhof's law has been postulated to hold in the $x=L$ center of the graph with the wedges oriented, conventionally, inwards,

$$
\begin{gathered}
\psi_{j}(L)=\psi_{0}(L), \quad j=1, \ldots, q-1, \\
\sum_{j=0}^{q-1} \partial_{x_{j}} \psi_{j}(L)=0 .
\end{gathered}
$$

The simplification has been compensated by the "exotic' 17 external boundary conditions

$$
\begin{gathered}
\partial_{x_{j}} \psi_{j}(0)=i \alpha e^{\mathrm{i} j \varphi} \psi_{j}(0), \quad j=0,1, \ldots, q-1, \\
\varphi=2 \pi / q .
\end{gathered}
$$

\footnotetext{
${ }^{1}$ An anonymous referee of this paper correctly objected that these boundary conditions need not be considered exotic at all. The essence of such an objection may be found explained in paper 18 in which the authors show how, in the $q=2$ special case, these boundary conditions may be given a natural and elementary physical interpretation in terms of a perfecttransmission external scattering. Naturally, the presence of the additional phase factors still leaves the net inflow into the whole graph equal to zero at any $q>2$. Another extremely interesting aspect of the models of similar class has also been pointed out and discussed (though not yet published) by Stefan Rotter et al [19]
}

In the units $L=1$ we arrived at the $q=6$ secular equation

$$
\frac{k^{6}-\alpha^{6} \tan ^{4} k}{k^{6}+\alpha^{6} \tan ^{6} k} \tan k=0 .
$$

This equation admits the straightforward graphical solution which is being sampled here via Figs. 2 and 3 .

A thorough inspection of these two pictures reveals that there exists the so called Kato's [20] exceptionalpoint value of the critical coupling which may be localized to lie at $\alpha_{\min } \approx 0.7862806298$. At this value of the coupling (which is, in our model, real) the number of the real roots of the secular determinant changes. More precisely, the more detailed Fig. 2 shows that at the not too large value of coupling $\alpha=7 / 10$ there exist four distinct real zeros of the secular determinant in the interval of $k \in(0,2)$. The subsequent inspection of Fig. 3 enables us to find out that after the transition to an overcritical value of $\alpha=1$ the two lowest roots disappeared and, obviously, complexified (in an independent calculation, the position of these "missing roots" in the complex plane of $k$ has been localized by an ad hoc, brute-force numerical method).

\section{Summary AND OUTLOOK}

The idea of the study of the non-Hermitian starshaped quantum graphs relates, equally strongly, to the studies of its non-Hermitian predecessors which remain topologically trivial (cf. their samples, say, in [2,4]) and to the studies of the topologically nontrivial, genuine $q$-pointed-star-shaped quantum graphs with $q>2$ where the Hamiltonian $H$ of the quantum system is assumed self-adjoint in one of the most common Hilbert-space forms of square-integrable wave functions (see, pars pro toto, an overall discussion of this approach in recent paper [21] which lists also further references in this area). Each of these predecessors 
offers a slightly different motivation which originated much more from the needs of the phenomenology and quantum theory in the former case (cf., in particular, [2]) and which was able to make use of an extensive knowledge of the underlying mathematics and, in particular, of the existing theorems and results of functional analysis in the latter context.

In this sense, at present as well as in the nearest future, the combination of the two ideas may be expected to share both their merits and weaknesses. In particular, the strengths may be expected to emerge due to the related new perspectives in the applications in physics (cf. e.g., the new perspective which appeared opening in the experimental optics using newly developed anomalous metamaterials [22]). At the same time, the most important weak points of the theory may, understandably, be identified with the current lack of the reliable abstract mathematical understanding of some subtleties which naturally emerge not only in connection with the possible, uninhibited encounter of the Kato's exceptional points, say, in the space of couplings, but also in connection with our up to now fairly vague - current understanding of the representation theory of Hilbert spaces in which the operator of metric is admitted non-trivial (cf., e.g., 23, 24] for further reading).

\section{ACKNOWLEDGEMENTS}

The support by the GAČR grant P203/11/1433 is acknowledged. The author also appreciates discussions with Ondrej Turek and Taksu Cheon (Kochi University), with Sergii Kuzhel (AGH University in Cracow) and, last but not least, unidirectionally, with anonymous referees.

\section{ReFERENCES}

[1] P. Exner, J. P. Keating, P. Kuchment and A. Teplyaev, Analysis on Graphs and Its Applications (AMS, Rhode Island, 2008).

[2] C. M. Bender, Rep. Prog. Phys. 70 (2007) 947.

[3] M. Znojil, SIGMA 5, 001 (2009), arXiv:0901.0700.

[4] J. Železný, The Krein-space theory for non-Hermitian PT-symmetric operators (MSc thesis, FNSPE CTU, 2011);
[5] P. Siegl, Non-Hermitian quantum models, indecomposable representations and coherent states quantization $(\mathrm{PhD}$ thesis, Univ. Paris Diderot \& FNSPE CTU, 2011).

[6] M. Znojil, Acta Polytechnica 50 (2010) 62.

[7] J. Dieudonne, Proc. Int. Symp. Lin. Spaces (Pergamon, Oxford, 1961), p. 115.

[8] F. G. Scholtz, H. B. Geyer and F. J. W. Hahne, Ann. Phys. (NY) 213 (1992) 74.

[9] P. Dorey, C. Dunning and R. Tateo, J. Phys. A: Math. Theor. 40 (2007) R205 and Pramana - J. Phys. 73 (2009) 217.

[10] A. Mostafazadeh, Int. J. Geom. Meth. Mod. Phys. 7 (2010) 1191.

[11] M. Znojil, Phys. Rev. D. 80 (2009) 045022.

[12] M. Znojil, Phys. Rev. D. 80 (2009) 105004.

[13] P. Exner and K. Němcová, J. Phys. A: Math. Gen. 36 (2003) 10173.

[14] M. Znojil, J. Phys. A: Math. Theor. 43 (2010) 335303; M. Znojil, Int. J. Theor. Phys. 50 (2011) 1052 and 1614.

[15] M. Znojil, Can. J. Phys. 90 (2012) 1287.

[16] M. Znojil, Phys. Rev. D. 80 (2009) 045009.

[17] M. Znojil, SIGMA 4, 001 (2008), arXiv:0710.4432v3

[18] H. Hernandez-Coronado, D. Krejcirik and P. Siegl, Phys. Lett. A 375 (2011) 2149.

[19] S. Rotter, "Exceptional points in open and closed gain-loss structures", http://phhqp11.in2p3.fr/ Wednesday_29_files/RotterSL12.pdf

[20] T. Kato, Perturbation theory for linear operators (Springer, Berlin, 1966).

[21] L. P. Nizhnik, Methods Funct. Anal. Topology 18 (2012) 68.

[22] C. E. Rüter, R. Makris, K. G. El-Ganainy, D. N. Christodoulides, M. Segev and D. Kip, Nat. Phys. 6 (2010) 192.

[23] F. Bagarello and M. Znojil, J. Phys. A: Math. Theor. 45 (2012) 115311; A. Mostafazadeh, Phil. Trans. R. Soc. A 371 (2013) 20120050.

[24] P. Siegl and D. Krejcirik, Phys. Rev. D 86 (2012) 121702(R). 